\title{
Vortex shear effects in layered superconductors
}

\author{
V. Braude and A. Stern \\ Department of Condensed Matter Physics, The Weizmann Institute of Science, Rehovot 76100, Israel
}

(November 7, 2018)

\begin{abstract}
Motivated by recent transport and magnetization measurements in BSSCO samples [B. Khaykovich et al., Phys. Rev B 61, R9261 (2000)], we present a simple macroscopic model describing effects of inhomogeneous current distribution and shear in a layered superconductor. Parameters of the model are deduced from a microscopic calculation. Our model accounts for the strong current non-linearities and the re-entrant temperature dependence observed in the experiment.
\end{abstract}

\section{INTRODUCTION}

Transport measurements are widely used in studies of vortex dynamics of high- $T_{c}$ superconductors. When the current distribution in the sample is not homogeneous, the results of the measurements are usually interpreted in terms of a local resistivity tensor. Due to high anisotropy of these materials the in-plane resistivity $\rho_{x y}$ is much smaller than the out-of-plane resistivity $\rho_{z}$. Commonly the resistivity is assumed to be a local function of the current density, and to depend on the applied magnetic field and the temperature ${ }^{2-4}$. A recent experiment by Khaykovich et al. ${ }^{1}$ does not fit into this scheme. In this experiment transport and magnetization measurements
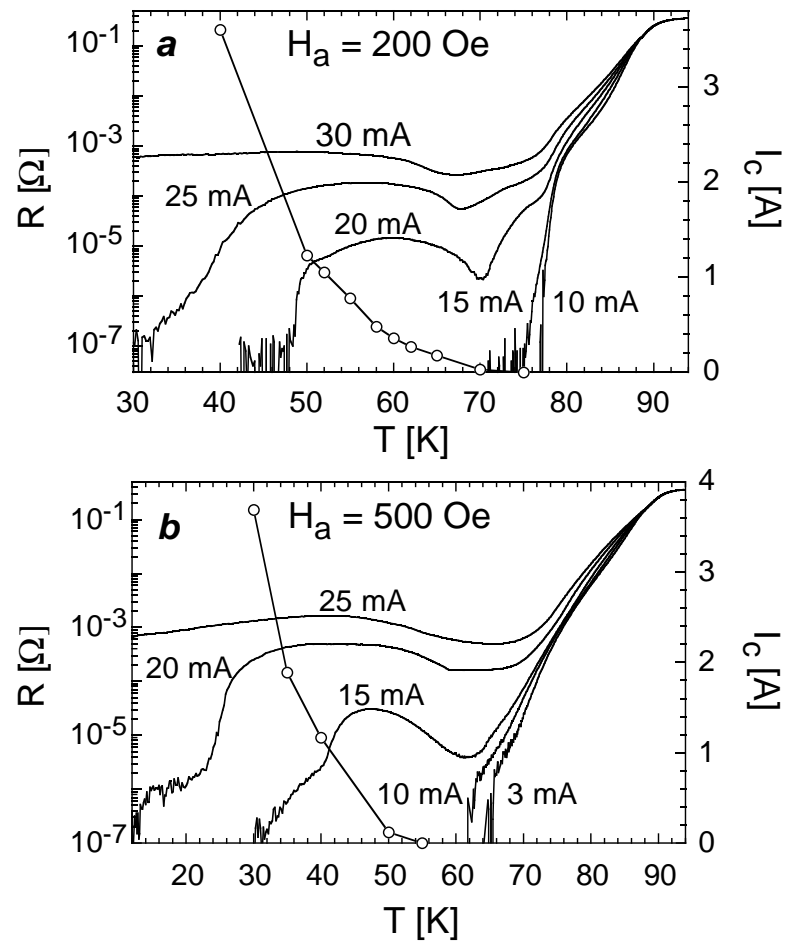

FIG. 1. Resistance at various $I_{a}$ (left axis, log scale) and magnetically measured critical current (right axis, linear scale, open circles) vs $\mathrm{T}$ for the irradiated sample, $H_{a}=200$ Oe (a) and $H_{a}=500$ Oe (b) (taken from Khaykovich et al. ${ }^{1}$ ) in BSCCO crystals at elevated transport currents and perpendicular magnetic field are performed, using high quality BSCCO platelets with current leads attached to the top surface and an array of 2DEG Hall sensors to the bottom surface. At a first glance, the pictures that emerge from the transport and the magnetization measurements are mutually contradicting. Transport measurements reveal finite resistivity below the magnetic irreversibility line, in the superconducting state. This resistivity is non-monotonic with temperature, showing reentrant behavior, and non-linear with current. As seen in the graphs of $R$ vs. $T$, Fig. 1, at low transport currents $R(T)$ is monotonic, dropping below experimental resolution when temperature is reduced. At elevated currents, the resistance initially drops as $T$ is lowered, but then goes up, the bump being steeper at lower currents. Also $R(T)$ shows strong non-linearity, so that an increase of the current by $30 \%$ or less may result in enhancement of $R$ by orders of magnitude. The source of this resistance is, presumably, vortex flow as a response to the electric current.

In contrast, local magnetization measurements in the presence of transport current, shown in Fig. 2a, indicate that the vortices are pinned. These measurements can be well described in terms of the Bean model of the critical state $^{6,7}$. The model states that below the irreversibility line the local current density equals either zero, or the critical current density, directed in such a way as to obtain the total transport current and the magnetization. The spatial distribution of the magnetic field is then given by the Biot-Savart law ${ }^{8}$. Since the current density nowhere exceeds the critical one, the Bean model predicts zero resistance. Within the Bean model finite resistivity can be expected only above the magnetically measured irreversibility line, which in Fig. 2a occurs above 1600 Oe. Indeed, at low $I_{a}$ the measurements (carried below the irreversibility line) show practically zero resistance. However, at elevated currents, substantial resistance is measured concurrently with the hysteretic magnetization well below the irreversibility line, as seen in Fig. 2a. Figure $2 \mathrm{~b}$ shows the corresponding field profile $B_{z}(x)$, obtained by the array of Hall sensors at 400 Oe in presence of transport current on increasing and decreasing $H_{a}$. A clear Bean profile is observed. Fitting this profile to the 

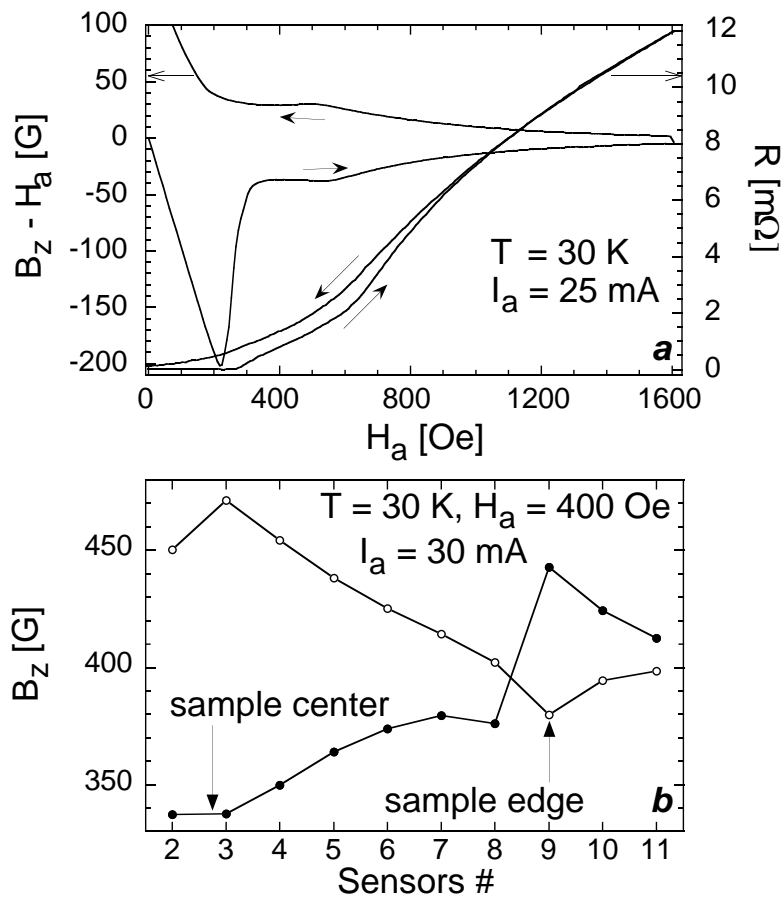

FIG. 2. (a) Resistance (right axis) and hysteretic magnetization loop in the sample center (left axis) vs. $H_{a}$ at $T=30 \mathrm{~K}$ and $I_{a}=25 \mathrm{~mA}$. (b) Profile of magnetic induction across the sample at 400 Oe on increasing $(\bullet)$ and decreasing (o) fields (taken from Khaykovich et al. ${ }^{1}$ ).

theoretical field distribution in platelet sample results in total critical current of $I_{c}=4.2 \mathrm{~A}$, which is more than two orders of magnitude higher than the transport current of $25 \mathrm{~mA}$. Figure 1 shows $I_{c}(T)$ determined from the Bean profiles together with the resistive data. The re-entrant resistance always occurs in the region where zero resistance is expected, since the transport current is much lower than the critical current.

Thus, the main puzzling observations of Khaykovich et $a l$. are the nonvanishing resistance below the irreversibility line, which indicates flux flow, coexisting with magnetization measurements which indicate that the vortices are pinned, the re-entrant behavior of the resistance with the temperature and its strongly nonlinear dependence on the current.

Khaykovich et al. ${ }^{1}$ suggest the following qualitative understanding of the observation. BSCCO, being a strongly anisotropic type II high $T_{c}$ superconducting material, consists of superconducting $\mathrm{CuO}_{2}$ layers, separated by insulating barriers. Each layer can carry current, resulting in total parallel current along the sample. Also, due to Josephson coupling between the layers, current can flow perpendicular to the layers. Because of large anisotropy a typical ratio of the perpendicular and parallel resistivities is $\simeq 10^{4}$ in the normal state. In perpendicular magnetic field the flux penetrates the system in form of vortices, but, due to weak interlayer coupling, these are two dimensional "pancakes", rather than three dimensional filaments. Pancakes in the same layer repel one another, while those in different layers attract via Josephson and magnetic coupling ${ }^{7}$. In the experiment, the leads are attached to the top surface of the crystal. Hence the current distribution is non-homogeneous along the sample thickness, planes near the bottom of the crystal carrying much lower current than those at the top. As temperature decreases, pinning of vortices becomes more effective. Eventually the critical current density exceeds current density near the bottom. Then pancake vortices at the bottom stop moving, while pancakes at the top maintain their high velocity, since current density there is much higher than the critical current density. As a result, velocity gradient of pancake motion between different layers is increased. This, in turn, leads to shear-induced phase slippage between the adjacent $\mathrm{CuO}_{2}$ planes, reducing the Josephson coupling and increasing the perpendicular resistance $\rho_{z}$. The larger $\rho_{z}$ causes the current to flow in a thinner part of the sample, thus making the process self-enhancing. Since all of the transport current flows in a few layers near the top of the sample, finite resistance exists at currents much lower than the critical current expected from the Bean model. Magnetization measurements, on the other hand, measure the magnetic response of all layers. When the vortices are pinned in most layers, this response is irreversible.

In this work we take this qualitative explanation as a starting point and construct macroscopic and microscopic models to analyze the experiment. We start by presenting a macroscopic model in which the sample is assumed to be constructed of a resistive part, an interface and a dissipationless part. The perpendicular resistivity of the resistive part is assumed to depend on "vortex shear". The parameters of this model are introduced phenomenologically. We then examine the dependence of the sample's resistance on these parameters, and the conclusions that may be drawn regarding the dependence of the resistance on the temperature and current. Following that we construct a microscopic model aimed at deriving an expression relating the conductivity in the direction perpendicular to the layers to the inter-layer variation of the current parallel to the layers. Finally we compare the conclusions of our model to the experimental findings. Although we find a general agreement, we also point out some remaining difficulties, associated mostly with the lack of quantitative information regarding several of the parameters of the model.

\section{THE MACROSCOPIC MODEL}

As we focus here on the consequences of inhomogeneity in the current distribution in $z$ direction, we use a one-dimensional model in which all quantities can vary only in this direction. Since scales of interest are much larger than the microscopic scale defined by the spacing between adjacent superconducting layers, we take a continuous limit in $z$ direction. 


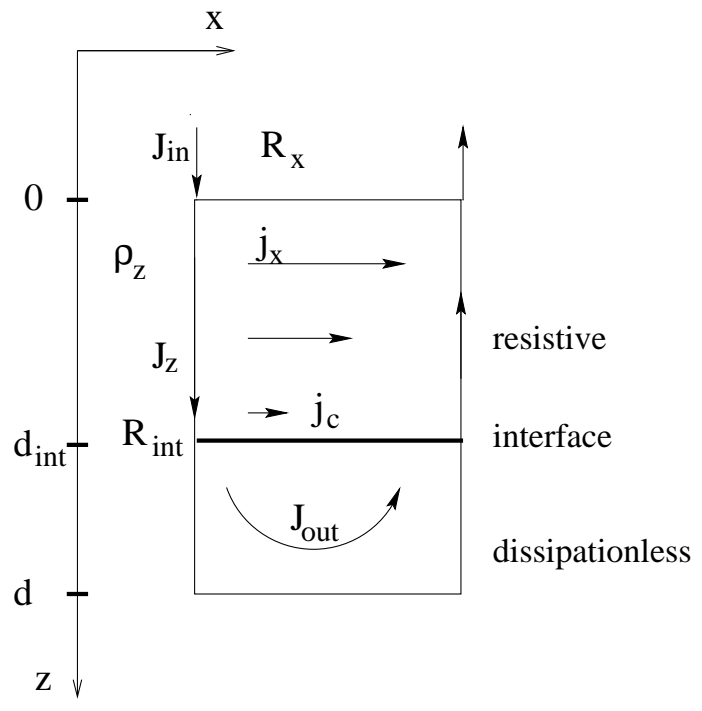

FIG. 3. The macroscopic model.

The model is described as follows. A current $J_{i n}$ is injected into a system of depth $d$ from above. Part of the current then flows horizontally as $j_{x}$ and the rest - vertically down as $J_{z}$ (then, of course, returning vertically up at the other end of the system). Below the depinning temperature $T_{d}$, when there is non-zero critical current density $j_{c}$, the system can be divided into two parts, by the value of the in-plane current $j_{x}$. The upper part of the system carries current density larger than $j_{c}$, so it has finite resistance, while at the lower part the current density is smaller than $j_{c}$, and thus it has zero resistance. Accordingly, we consider the system as consisting of two phases: a resistive phase at the top, having parallel resistivity $R_{x}$ and perpendicular resistivity $\rho_{z} / 2$, and a dissipationless phase with zero resistivity. Note, that since the current first flows down and then up, the total perpendicular resistivity it experiences is $\rho_{z}$. Furthermore, we assume that current crossing the interface between the two phases faces a resistance $R_{\text {int }} / 2$. The position of the interface is determined by the condition $j_{x}=j_{c}$. This condition also fixes the current $J_{\text {out }}$ flowing through the dissipationless region:

$$
J_{\text {out }} R_{\text {int }}=j_{c} R_{x} .
$$

At high temperatures $j_{c}$ is zero, and the system consists only of the dissipative phase.

The basic equations governing the distribution of the current in the dissipative phase are the two Kirchoff equations. The continuity equation is (note that in the geometry we consider $J_{z}$ and $j_{x}$ have different dimensions, since $J_{z}$ is a two dimensional current density, while $j_{x}$ is a three dimensional current density):

$$
\partial_{z} J_{z}+j_{x}=0
$$

and the equation giving the total voltage is:

$$
V=\int_{0}^{z} J_{z}\left(z^{\prime}\right) \rho_{z}\left(z^{\prime}\right) d z^{\prime}+j_{x}(z) R_{x}
$$

As we show below in the microscopic analysis, the $z$-axis resistivity depends on the difference between $j_{x}$ in adjacent layers $\partial_{z} j_{x}$, and this dependence may be approximated by

$$
\rho_{z}=\rho_{0}+\sqrt{\rho_{1}^{2}+\left(f \partial_{z} j_{x}\right)^{2}}=\rho_{0}+\sqrt{\rho_{1}^{2}+\left(f \partial_{z}^{2} J_{z}\right)^{2}},
$$

while $R_{x}$ is assumed to be a constant parameter. The term $f \partial_{z} j_{x}$ in the resistivity $\rho_{z}$ is a contribution of the "shear" between vortices in different layers to the out-ofplane resistance. It originates from the effect of a velocity gradient between vortices in adjacent planes on the Josephson coupling between the planes.

Substituting Eq. (2) into Eq. (3) and differentiating with respect to $z$ we obtain:

$$
J_{z}\left(\rho_{0}+\sqrt{\rho_{1}^{2}+\left(f \partial_{z}^{2} J_{z}\right)^{2}}\right)-R_{x} \partial_{z}^{2} J_{z}=0 .
$$

This equation can be solved only if the condition

$$
J_{z}<\frac{R_{x}}{\rho_{0}} \partial_{z}^{2} J_{z}
$$

is satisfied. Designating $J \equiv J_{z}, J^{\prime \prime} \equiv \partial_{z}^{2} J_{z}$ and solving for $J^{\prime \prime}$, we obtain

$$
\begin{aligned}
J^{\prime \prime}= & \frac{1}{\left(R_{x} / J\right)^{2}-f^{2}} \\
& \times\left(R_{x} \rho_{0} / J \pm \sqrt{\left(R_{x} \rho_{1} / J\right)^{2}+f^{2}\left(\rho_{0}^{2}-\rho_{1}^{2}\right)}\right) .
\end{aligned}
$$

The condition (6) requires that plus sign be taken in Eq. (7) and that $J<J_{0} \equiv R_{x} / f$. This means that as $J_{\text {in }} \rightarrow J_{0}$, both $J^{\prime \prime}$ and $J^{\prime}$ diverge, so that the voltage $V$ also diverges, and the system becomes insulating. In fact, as $J_{i n} \rightarrow J_{0}$, current gradients in the system become large, and then the quasi-particle channel for $z$-axis currents needs to be taken into account, as analyzed below. When doing this, we find that $J_{0}$ is actually not a cutoff value for the injected current, but rather a parameter that signifies the importance of shear effects. Thus, when $J_{\text {in }}$ becomes comparable with $J_{0}$, shear becomes strong, and the resistance is strongly non-linear with $J_{i n}$.

Substituting the solution for $J^{\prime \prime}$ into Eq. (4), the perpendicular resistivity can be expressed in terms of $J$ :

$$
\rho_{z}=\rho_{0} \frac{1+\sqrt{r^{2}+\kappa^{2}\left(1-r^{2}\right)}}{1-\kappa^{2}},
$$

where we used reduced quantities $\kappa \equiv J / J_{0}$ and $r \equiv$ $\rho_{1} / \rho_{0}$. It is plotted in Fig. 4. Again, this is valid for $J$ not too close to $J_{0}$.

It is possible to integrate Eq. (7). Some intuition to it may be obtained by noticing that Eq. (7) may be viewed as an equation of motion for a particle whose one dimensional coordinate is $J$, its "time" is $z$, and the potential 


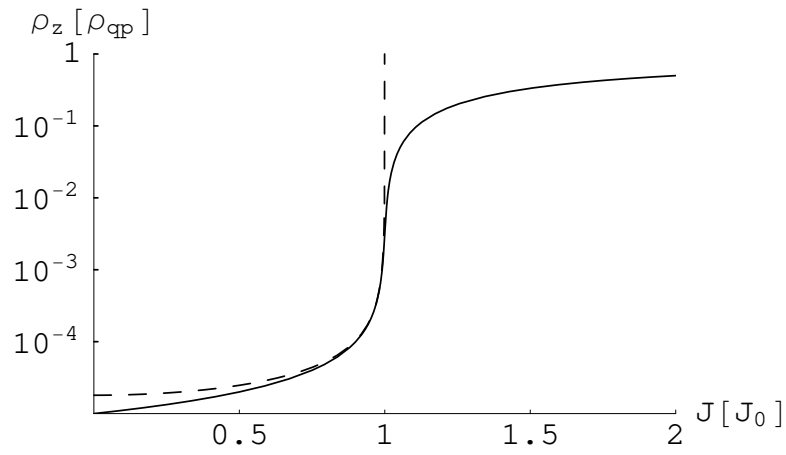

FIG. 4. Perpendicular resistivity $\rho_{z}$ for the model without quasiparticle channel, Eq. (8) (dashed line) and with quasiparticle channel, Eq. (12) (solid line).

it moves in is

$$
\begin{aligned}
U(J)=\frac{J_{0} \rho_{0}}{f}( & \sqrt{r^{2}+\kappa^{2}\left(1-r^{2}\right)} \\
& \left.+\ln \left|1-\sqrt{r^{2}+\kappa^{2}\left(1-r^{2}\right)}\right|\right)
\end{aligned}
$$

This potential is plotted in Fig. 5 .

The analysis leading to Eq. (7) neglects inter-layer current flow by means of quasi-particle tunneling. When the current gradient $J^{\prime \prime}$ gets large, $\rho_{z}$ becomes large, and a large portion of the current flows perpendicularly in the form of quasiparticles. Hence in this high-gradient limit the perpendicular resistivity should be modeled by two resistors in parallel. Also, since in this regime the current gradients are large, a linearized expression for the Josephson channel resistivity, Eq. (4), can be used. Thus the Josephson channel carries a resistivity $\rho_{0}+f \partial_{z} j_{x}$, while the quasiparticle channel's resistivity is $\rho_{q p}$. The total perpendicular resistivity is

$$
\rho_{z}^{-1}\left(\partial_{z} j_{x}\right)=\left(\rho_{0}+f \partial_{z} j_{x}\right)^{-1}+\rho_{q p}^{-1}
$$

It is assumed, of course, that $\rho_{q p} \gg \rho_{0}$. Using this assumption and solving again for the current distribution,

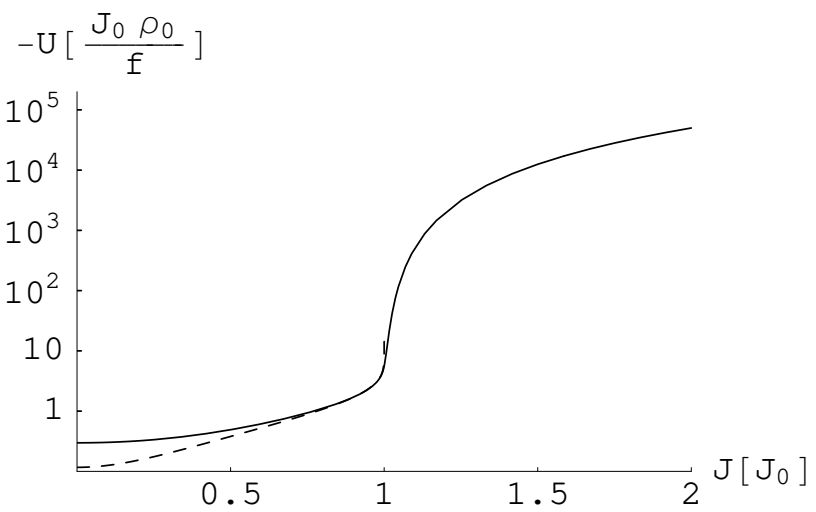

FIG. 5. Potential $U(J)$ (taken with minus sign) for the model without quasiparticle channel, Eq. (9) (dashed line) and with quasiparticle channel, Eq. (13) (solid line). we get a differential equation

$$
J^{\prime \prime}=\frac{\rho_{q p}}{2 f}\left(\kappa-1 \pm \sqrt{(\kappa-1)^{2}+4 \kappa \rho_{0} / \rho_{q p}}\right),
$$

where again $\kappa \equiv J / J_{0}$. In order to have $J^{\prime \prime}>0$, we need to choose the plus sign. The perpendicular resistivity can be expressed in terms of $J$ :

$$
\rho_{z}=\rho_{q p} \frac{\kappa-1+\sqrt{(\kappa-1)^{2}+4 \kappa r_{2}}}{2 \kappa},
$$

where $r_{2} \equiv \rho_{0} / \rho_{q p}$. It is plotted in Fig. 4 .

Integrating Eq. (11), we obtain the corresponding "potential", plotted in Fig. 5:

$$
\begin{aligned}
& U(J)=-\frac{J_{0} \rho_{q p}}{4 f} \\
& \times\left[(\kappa-1)^{2}+4 r_{2}\left(1-r_{2}\right) \operatorname{arcsh} \frac{\kappa-1+2 r_{2}}{2 \sqrt{r_{2}\left(1-r_{2}\right)}}\right. \\
& \left.\quad+\left(\kappa-1+2 r_{2}\right) \sqrt{4 r_{2}\left(1-r_{2}\right)+\left(\kappa-1+2 r_{2}\right)^{2}}\right] .
\end{aligned}
$$

In both cases we may use $U(J)$ together with the boundary conditions to determine the resistance of the system. The "velocity" of the particle is given by

$$
\partial_{z} J_{z}=-\sqrt{2[C-U(J)]}
$$

Here $C$ is a constant determined by the boundary conditions, which require $J_{z}(z=0)=J_{i n}$, and either $J_{z}(d)=0$, in the case where the whole sample is resistive so that $j_{x}>j_{c}$; or $J_{z}\left(d_{\text {int }}\right)=J_{\text {out }}$ and $\partial_{z} J_{z}\left(d_{\text {int }}\right)=j_{c}$, for the case where the lower part of the sample is dissipationless and is separated from the upper part by an interface at depth $d_{\text {int }}$. The first case takes place at temperatures above $T_{d}$, where $j_{c}=0$, while the second case - when the temperature is below $T_{d}$. For the second case the derivative $J^{\prime}(z=0)$ can be found:

$$
J^{\prime}(0)=-\sqrt{2\left(U\left(J_{\text {out }}\right)-U\left(J_{\text {in }}\right)\right)+j_{c}^{2}} .
$$

Finally, after solving for the current profile $J(z)$ we may calculate the resistance of the sample to be

$$
R=\frac{V}{J_{i n}}=-\frac{R_{x} J^{\prime}(0)}{J(0)} .
$$

We now summarize how the resistance depends on various parameters of the model.

\section{A. Dependence on the injected current $J_{i n}$}

For high temperatures, when the whole sample is dissipative, the resistance increases monotonously with $J_{i n}$, experiencing a sharp increase around $J_{0}$. This is because 
larger currents produce larger current gradients, which, in turn, increase the vertical resistivity $\rho_{z}$.

The low temperature case, where there is an interface, is more complicated and depends on the value of the interface resistance $R_{\text {int }}$. To investigate the dependence of the resistance on the current, we need to differentiate the expression

$$
R=-\frac{R_{x} J^{\prime}(0)}{J_{\text {in }}}=\frac{R_{x} \sqrt{2\left(U\left(J_{\text {out }}\right)-U\left(J_{\text {in }}\right)\right)+j_{c}^{2}}}{J_{\text {in }}}
$$

with respect to $J_{i n}$. We have:

$$
\begin{aligned}
\frac{d R}{d J_{i n}} & =-\frac{R_{x}}{J_{i n}} \frac{d J^{\prime}(0)}{d J_{i n}}+R_{x} \frac{J^{\prime}(0)}{J_{i n}^{2}} \\
& =-\frac{R_{x}}{J_{i n} J^{\prime}(0)} \frac{d U\left(J_{i n}\right)}{d J_{i n}}+R_{x} \frac{J^{\prime}(0)}{J^{2}} .
\end{aligned}
$$

Substituting $d U(J) / d J=J^{\prime \prime}$ and multiplying by a positive quantity $-R_{x} J^{\prime}(0)$, we obtain

$$
\frac{d R}{d J_{i n}} \propto \frac{R_{x}^{2} J^{\prime \prime}(0)}{J_{\text {in }}}-R^{2}\left(J_{i n}\right)
$$

The above derivative is definitely positive at $J_{i n} \approx J_{0}$, since, as we saw above, the Cooper pair channel gets blocked, and the resistance of the system rises abruptly as $J_{\text {in }}$ approaches $J_{0}$. Hence it is only left to determine the dependence on $J_{i n}$ for $J_{i n}<<J_{0}$. For this case we can neglect the quasi-particle contribution and use the expression (7) for $J^{\prime \prime}(0)$. After a minor manipulation we obtain

$$
\frac{d R}{d J_{i n}} \propto \frac{R_{x} \rho_{0}\left(1-r^{2}\right)}{1-\sqrt{r^{2}+\kappa_{i n}^{2}\left(1-r^{2}\right)}}-R^{2}\left(J_{i n}\right)
$$

where $\kappa_{\text {in }}=J_{\text {in }} / J_{0}$.

The sign of this expression determines whether the resistance increases or decreases with the injected current $J_{i n}$. It is easily verified that this expression is increasing with $\kappa_{i n}$ (i.e. with $J_{i n}$ ). Hence it is enough to determine the sign at the smallest current at which the model is applicable, $J_{\text {in }}=J_{\text {out }}$ : if it is positive, then the resistance increases monotonically with the current, while if it is negative, the resistance first decreases and then starts to grow as the current becomes large enough, c.f. Fig. 6 . Substituting $\kappa_{\text {in }}=\kappa_{\text {out }}$ and $R\left(J_{\text {out }}\right)=R_{\text {int }}$, we get:

$$
\left.\frac{d R}{d J_{\text {in }}}\right|_{J_{\text {in }}=J_{\text {out }}} \propto \frac{R_{x} \rho_{0}\left(1-r^{2}\right)}{1-\sqrt{r^{2}+\left(j_{c} f / R_{\text {int }}\right)^{2}\left(1-r^{2}\right)}}-R_{\text {int }}^{2} .
$$

The result is a decreasing function of $R_{\text {int }}$. It is positive for small $R_{\text {int }}$ (which should be larger than $f j_{c}$ in order to satisfy $J_{\text {out }}<J_{0}$ ), negative for large $R_{\text {int }}$ and vanishes at $R_{\text {int }}=R_{\text {int }, 0}$, given by

$$
R_{\text {int }, 0}=\sqrt{R_{x} \rho_{0}+j_{c}^{2} f^{2} / 2+\sqrt{\left(R_{x} \rho_{0}+j_{c}^{2} f^{2} / 2\right)^{2}-R_{x}^{2} \rho_{0}^{2}\left(1-r^{2}\right)}} .
$$

Thus, the dependence of the resistance on the current is controlled by the value of $R_{\text {int }}$, as is seen in Fig. 6 . For $f j_{c}<R_{\text {int }}<R_{\text {int }, 0}$ the resistance increases monotonically with the current. But if $R_{\text {int }}>R_{\text {int }, 0}$, the resistance decreases for small currents $J_{\text {out }}<J_{\text {in }}<J_{1}$, where $J_{1}$ is the solution of an equation

$$
\frac{R_{x} \rho_{0}\left(1-r^{2}\right)}{1-\sqrt{r^{2}+\left(J_{1} / J_{0}\right)^{2}\left(1-r^{2}\right)}}=R^{2}\left(J_{1}\right) .
$$

The physical explanation for this behavior is that when the current is increased, the interface is pushed downwards, increasing the thickness of the upper (dissipative) layer. If the interface is highly conducting (small $R_{\text {int }}$ ), most of the current is shunted through the lower (dissipationless) part of the system, so the increase in the upper layer thickness increases the resistance of the system. However, if the interface is almost insulating (large $\left.R_{\text {int }}\right)$, most of the current flows through the upper part, and by increasing its thickness the resistance of the system is decreased. Of course, at large enough currents the rapid increase of $\rho_{z}$ due to shear has dominant effect, so the resistance increases anyway. As we show below, the relevant case is large $R_{\text {int }}$, when a re-entrant behavior as a function of the temperature takes place. Hence, below $T_{d}$ an increase in the current influences the system in two opposite ways: it tends to decrease the resistance by moving the interface downwards; while through the effect of shear it tends to increase it. Also, we see that a strong increasing dependence of the resistance on the

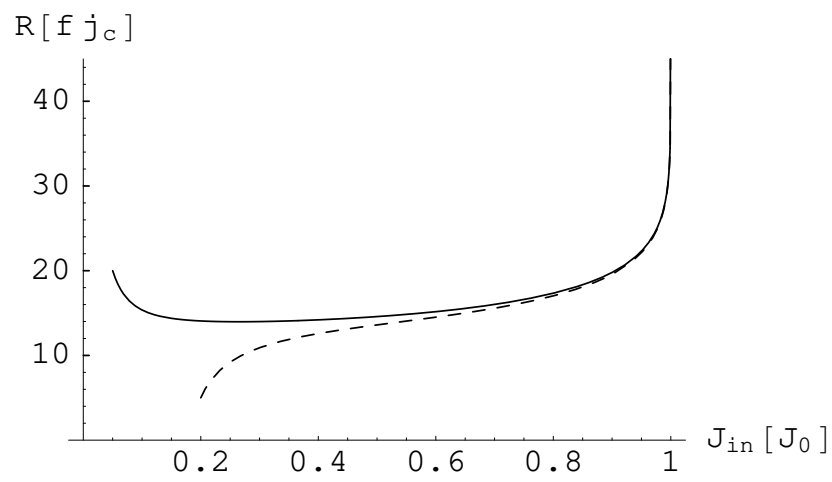

FIG. 6. Sample resistance $R\left(J_{i n}\right)$ for $R_{\text {int }}<R_{\text {int }, 0}$ (dashed line) and for $R_{i n t}>R_{\text {int }, 0}$ (solid line). 
current appears only when $J_{\text {in }} \approx J_{0}$, this being true bot above and below $T_{d}$.

The difference between the results given here and the qualitative arguments of Ref. [1] may be understood in the following qualitative way. Suppose that a current $J_{\text {in }}$ flows into the system and generates a current profile $J(z)$ with an interface at $z=d$. When $J_{\text {in }}$ is slightly increased one may expect the current gradient $\partial_{z} j_{x}$ to increase, thus increasing $\rho_{z}$, increasing anisotropy and pushing the interface upwards. The shear-induced increase in $\rho_{z}$ and the motion of the interface both tend then to increase the resistance. Our model yields a different picture: as $J_{i n}$ is increased, the interface is shifted downward, thus reducing the resistance. The motion of the interface and the shear-induced increase of $\rho_{z}$ operate then in opposite directions.

\section{B. Dependence on the intralayer resistivity $R_{x}$}

For the case when there is no interface in the system, increasing $R_{x}$ makes the current distribution more homogeneous, so that $j_{x}(0)$ and $j_{x}(d)$ differ less. Put in another way, $J_{0} \equiv R_{x} / f$ grows. Because of this, the effects of inter-layer vortex shear become weaker, and the vertical resistivity $\rho_{z}$ decreases. Hence the total resistance $R$ is influenced by two opposite effects: increase of $R_{x}$ directly increases $R$, this effect being dominant at small currents. On the other hand, through the decrease of $\rho_{z}$ it tends to decrease $R$, this effect becoming dominant at strong currents, when effect of shear is important. Hence the resistance grows with $R_{x}$ at small $J_{i n}$, while it decreases with $R_{x}$ as $J_{i n}$ approaches $J_{0}$.

\section{Dependence on the critical current $j_{c}$}

Next we discuss the dependence of the sample resistance on the critical current. We disregard a possible dependence of various parameters (like $R_{\text {int }}$, for instance) on $j_{c}$ and consider only a variation of $R$ due to a shift in the position of the interface and the subsequent current redistribution. To determine the sign of the derivative $\frac{\partial R}{\partial j_{c}}$, we use the expression given by Eq. (17). First, it is easy to see that the sign of the derivative is independent of the value of injected current $J_{i n}$. Hence we can find it at $J_{\text {in }}=J_{\text {out }}$ (i.e. when the interface is right at the top of the sample). But when this condition is satisfied, the resistance of the system is constant and equal to $R_{\text {int }}$. Thus,

$$
\frac{d R\left(J_{\text {in }}=J_{\text {out }}\right)}{d j_{c}}=\frac{\partial R}{\partial j_{c}}+\left.\frac{\partial R}{\partial J_{\text {in }}}\right|_{J_{\text {in }}=J_{\text {out }}} \frac{d J_{\text {out }}}{d j_{c}}=0 .
$$

Then, using Eq. (1), we see that

$$
\frac{\partial R}{\partial j_{c}}=-\frac{R_{x}}{R_{\text {int }}} \frac{\partial R}{\partial J_{i n}}
$$

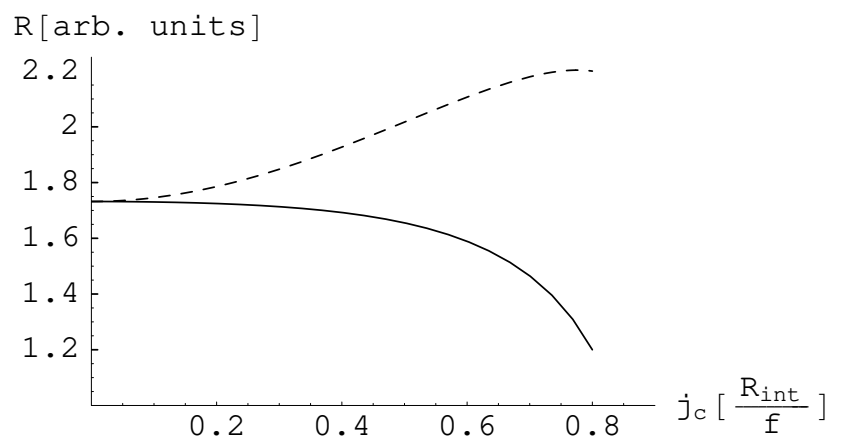

FIG. 7. Sample resistance $R\left(j_{c}\right)$ at $J_{i n}=0.8 J_{0}$

for $R_{\text {int }}>\sqrt{R_{x}\left(\rho_{0}+\rho_{1}\right)}$ (dashed line) and for $R_{\text {int }}<\sqrt{R_{x}\left(\rho_{0}+\rho_{1}\right)}$ (solid line).

Consequently, at small $J_{\text {in }}$ the dependence of $R$ on $j_{c}$ is opposite to its dependence on $J_{i n}$ (at larger currents the dependence on $j_{c}$ remains of the same type, while the dependence on $J_{\text {in }}$ may change, as was shown above). This behavior is natural, as by increasing $J_{i n}$ the interface is pushed downwards, while increasing $j_{c}$ it is pushed upwards. Hence citing the previous results we obtain that $R$ increases with $j_{c}$ for $R_{\text {int }}>R_{\text {int }, 0}$, while it decreases with $j_{c}$ for $R_{\text {int }}<R_{\text {int }, 0}$. Formulated in a different way, this means that $R$ increases with $j_{c}$ when $j_{c}<j_{c 0}$, where $j_{c 0}$ is given by

$$
j_{c 0}=\frac{R_{\text {int }}}{f} \sqrt{\frac{\left[1-R_{x} \rho_{0}\left(1-r^{2}\right) / R_{i n t}^{2}\right]^{2}-r^{2}}{1-r^{2}}} .
$$

In order that $j_{c 0}$ be real and positive, $R_{\text {int }}$ has to satisfy $R_{\text {int }}>\sqrt{R_{x}\left(\rho_{0}+\rho_{1}\right)}$, which is physically plausible, as the interface should be insulating enough in comparison to the resistive phase in order that the rise in its vertical position would increase the sample resistance. If this condition is not satisfied, or if $j_{c}>j_{c 0}, R$ decreases with $j_{c}$. Note that when $R_{\text {int }} \rightarrow \infty$ also $j_{c 0} \rightarrow \infty$, so that in this case $R$ increases with $j_{c}$ for any relevant value of $j_{c}$. The behavior of the resistance as a function of $j_{c}$ can be seen in Fig. 7. Also, from Eq. (17) we see that $\frac{d R}{d j_{c}} \sim \frac{1}{R}$ (since a square root is differentiated). Hence at large currents the dependence on $j_{c}$ becomes weaker. This is in contrast to the dependence on $J_{i n}$, which becomes very strong as $J_{i n} \rightarrow J_{0}$. All this, of course, is valid when $j_{c}$ is strong enough that there is an interface in the system.

\section{PERPENDICULAR RESISTIVITY DUE TO PARALLEL CURRENT GRADIENT}

The interplane transport properties of high- $T_{c}$ superconductors have been a subject of intense research over the past decade, both theoretical ${ }^{9,10}$ and 
experimental ${ }^{2,4,11-13}$. This transport, being of Josephson nature, is determined by the phase coherence between the adjacent layers. For a superconductor in a perpendicular magnetic field, the pancake vortex structure determines the above properties through the phase distribution. The vortex structure in high- $T_{c}$ superconductors exhibits a rich variety of phenomena, including decoupling, melting, pinning, Bose glass formation etc., due to thermal fluctuations ${ }^{14-17}$, point $\operatorname{defects}^{16-18}$ or columnar defects ${ }^{17,19-22}$. For the perpendicular resistivity $\rho_{z}$, the microscopic origin of the dissipation is less obvious than for the in-plane resistivity $\rho_{x}$, where it is understood in terms of the Lorentz force, acting on the pancakes. Koshelev ${ }^{10}$ proposed a microscopic mechanism for interplane dissipation, in which the pancake dynamics are shown to influence the interplane conduction, and calculated $\rho_{z}$ for the simplest case of non-interacting pancakes. Following Ref. 10 we analyze a simple microscopic model aimed at a derivation of a formula for a contribution to the resistivity in $\hat{\mathbf{z}}$ direction (perpendicular to the layers) $\rho_{z}^{3 D}$ of a superconducting slab due to a gradient in the current in $\hat{\mathbf{x}}$ direction (parallel to the layers). We first derive $\rho_{z}^{3 D}$ for a 3 -dimensional sample assuming no interactions between the vortex pancakes. Then we show how the results are modified in presence of interlayer and intralayer correlations between the pancake positions. Finally, we transform the 3 -D resistivity parameters into a form appropriate for the 1-dimensional model used in the previous section. That is, we show how $R_{x}, \rho_{0}$ and $f$ of the macroscopic model are derived from the resistivities of the 3 -dimensional model.

\section{A. Noninteracting pancakes}

We assume a layered superconductor with noninteracting pancake vortices in it. The vortices are mobile, and their relative diffusive motion provides a mechanism for perpendicular resistance. In addition, each layer carries a different current, causing different drift velocities of vortices in adjacent layers. This increases the decay of phase correlations in time, thus enhancing the perpendicular resistance.

We start from the Kubo formula for finite temperatures:

$$
\sigma_{z}^{3 D}=\frac{s j_{J}^{2}}{T} \int d \mathbf{r} d t\langle\sin \delta \phi(0,0) \sin \delta \phi(\mathbf{r}, t)\rangle .
$$

Here $s$ is the interplane separation, $j_{J}$ - the Josephson current and $\delta \phi$ - the gauge invariant phase difference between neighboring layers. We neglect interplane correlations, so that averages like $\langle\exp 1 \delta \phi\rangle$ are assumed to be zero and then

$$
\begin{aligned}
& \langle\sin \delta \phi(0,0) \sin \delta \phi(\mathbf{r}, t)\rangle \approx(1 / 2) \operatorname{Re}\langle\exp [1 S(\mathbf{r}, t)]\rangle, \\
& \text { where } S(\mathbf{r}, t) \equiv \delta \phi(\mathbf{r}, t)-\delta \phi(0,0) .
\end{aligned}
$$

Next we assume Gaussian randomness of $S$, so that

$$
\langle\exp [1 S(\mathbf{r}, t)]\rangle=\exp \left[-\left\langle S(\mathbf{r}, t)^{2}\right\rangle / 2\right]
$$

Thus we need to calculate the mean square of $S$. We write

$$
\begin{array}{r}
S(\mathbf{r}, t)=\sum_{i} \phi_{v}\left(\mathbf{r}-\mathbf{R}_{1, i}(t)\right)-\phi_{v}\left(\mathbf{r}-\mathbf{R}_{2, i}(t)\right) \\
-\phi_{v}\left(-\mathbf{R}_{1, i}(0)\right)+\phi_{v}\left(-\mathbf{R}_{2, i}(0)\right),
\end{array}
$$

where $\phi_{v}(\mathbf{r})$ is the phase distribution of a single vortex. Expanding, we write

$$
\begin{aligned}
S(\mathbf{r}, t)=\sum_{i} & {\left[\mathbf{r}-\Delta \mathbf{R}_{1, i}(t)\right] \nabla \phi_{v}\left(-\mathbf{R}_{1, i}\right) } \\
& -\left[\mathbf{r}-\Delta \mathbf{R}_{2, i}(t)\right] \nabla \phi_{v}\left(-\mathbf{R}_{2, i}\right),
\end{aligned}
$$

where $\Delta \mathbf{R}(t) \equiv \mathbf{R}(t)-\mathbf{R}(0)$ and

$$
\nabla \phi_{v}(\mathbf{r})=\frac{\hat{\mathbf{z}} \times \mathbf{r}}{r^{2}} .
$$

Now we assume that the pancakes in the layers are randomly placed, so that

$$
\begin{aligned}
\left\langle\nabla \phi_{v}\left(-\mathbf{R}_{1}\right) \nabla \phi_{v}\left(-\mathbf{R}_{2}\right)\right\rangle & =0 \quad \text { and } \\
\left\langle\nabla \phi_{v}\left(-\mathbf{R}_{1, i}\right) \nabla \phi_{v}\left(-\mathbf{R}_{1, j}\right)\right\rangle & =\delta_{i, j}\left\langle\nabla \phi_{v}\left(-\mathbf{R}_{1, i}\right)^{2}\right\rangle .
\end{aligned}
$$

Then the square of a sum breaks into a sum of squares, so that

$$
\begin{aligned}
\left\langle S(\mathbf{r}, t)^{2}\right\rangle=\sum_{i} & \left\langle\left(\left[\mathbf{r}-\Delta \mathbf{R}_{1, i}(t)\right] \nabla \phi_{v}\left(-\mathbf{R}_{1, i}\right)\right)^{2}\right\rangle \\
& +\left\langle\left(\left[\mathbf{r}-\Delta \mathbf{R}_{2, i}(t)\right] \nabla \phi_{v}\left(-\mathbf{R}_{2, i}\right)\right)^{2}\right\rangle .
\end{aligned}
$$

Now we can write for each layer

$$
\Delta \mathbf{R}(t) \approx \mathbf{v} t+\delta \mathbf{R}(t)
$$

where $\mathbf{v}$ is the drift velocity of vortices due to the current, and $\delta \mathbf{R}(t)$ is the diffusion term. It gives the main contribution at zero current gradient, and we will copy it from the Koshelev's article. Using the expression (32) we write

$$
\begin{aligned}
\left\langle S(\mathbf{r}, t)^{2}\right\rangle= & \sum_{i}\left(\mathbf{r}-\mathbf{v}_{1} t\right)^{2}\left\langle\left[\frac{\mathbf{R}_{x ; 1, i}}{R_{1, i}^{2}}\right]^{2}\right\rangle \\
& +\left(\mathbf{r}-\mathbf{v}_{2} t\right)^{2}\left\langle\left[\frac{\mathbf{R}_{x ; 2, i}}{R_{2, i}^{2}}\right]^{2}\right\rangle+\left\langle S_{\text {diff }}(t)^{2}\right\rangle .
\end{aligned}
$$

Now we calculate the averages:

$$
\begin{aligned}
\sum_{i}\left\langle\left[\frac{\mathbf{R}_{x}}{R^{2}}\right]^{2}\right\rangle & =\sum_{i} \frac{1}{2}\left\langle\frac{1}{R^{2}}\right\rangle=\frac{n}{2} \int \frac{d \mathbf{R}}{R^{2}} \\
& =\pi n \ln \frac{R_{\max }}{R_{\min }}
\end{aligned}
$$

where $n$ is the density of the vortices and $R_{\min }$ and $R_{\max }$ - the lower and upper cutoff radii. Substituting this, we obtain 


$$
\begin{aligned}
\left\langle S(\mathbf{r}, t)^{2}\right\rangle & =\left[\left(\mathbf{r}-\mathbf{v}_{1} t\right)^{2}+\left(\mathbf{r}-\mathbf{v}_{2} t\right)^{2}\right] \pi n \ln \frac{R_{\max }}{R_{\min }}+\left\langle S_{\text {diff }}^{2}(t)\right\rangle \\
& =\left[2(\mathbf{r}-\mathbf{V} t)^{2}+(\Delta \mathbf{v} t)^{2} / 2\right] \pi n \ln \frac{R_{\max }}{R_{\min }}+\left\langle S_{\text {diff }}^{2}(t)\right\rangle,
\end{aligned}
$$

where

$$
\mathbf{V}=\left(\mathbf{v}_{1}+\mathbf{v}_{2}\right) / 2 \text { and } \Delta \mathbf{v}=\mathbf{v}_{1}-\mathbf{v}_{2} .
$$

Substituting this result back into Eq. (27) and using Koshelev's result for $S_{\text {diff }}$, we obtain:

$$
\begin{aligned}
\sigma_{z}^{3 D}(\Delta v) & =\frac{s j_{J}^{2}}{2 T} \int_{t>0} d \mathbf{r} d t \exp \left(-\left[(\mathbf{r}-\mathbf{V} t)^{2}+(\Delta \mathbf{v} t)^{2} / 4\right] \pi n \ln \frac{R_{\max }}{R_{\min }}-2 \pi n D t \ln \left(R_{J}^{2} / R_{\text {min }}^{2}\right)\right) \\
& =\frac{s j_{J}^{2}}{2 T} \frac{1}{n \ln \left(R_{J} / a_{0}\right)} \frac{2}{\Delta v \sqrt{\pi n \ln \left(R_{J} / a_{0}\right)}} F\left(4 D \sqrt{\pi n \ln \left(R_{J} / a_{0}\right)} / \Delta v\right),
\end{aligned}
$$

where $D$ is the diffusion constant of pancake motion inside the layers. We used the Josephson radius $R_{J}$ for the upper cutoff radius and the average intervortex spacing $a_{0}$ - for the lower cutoff. The function $F(y)$ is defined by

$$
F(y) \equiv \int_{0}^{\infty} d x e^{-x^{2}-2 x y}=e^{y^{2}} \int_{y}^{\infty} d x e^{-x^{2}}=\frac{\sqrt{\pi}}{2} e^{y^{2}}[1-\operatorname{Erf}(y)]
$$

This function can be easily approximated for small and large values of its argument:

$$
F(y) \rightarrow\left\{\begin{array}{cc}
\frac{\sqrt{\pi}}{2}-y & \text { for } \quad y \ll 1, \\
\frac{1}{2 y}-\frac{2}{(2 y)^{3}} & \text { for } y \gg 1
\end{array} .\right.
$$

Using this and expressing the vortex velocity difference in terms of parallel current gradient modulus,

$$
\Delta v=\mu s^{2}\left(\Phi_{0} / c\right) \partial_{z} j_{x}^{3 D},
$$

where the average pancake mobility $\mu$ is connected with the diffusion constant $D$ by the Einstein relation $D=\mu T$, we obtain for the perpendicular resistance

$$
\rho_{z}^{3 D}\left(\partial_{z} j_{x}^{3 D}\right)=\frac{1}{j_{J}^{2}} \frac{2 D s\left(\Phi_{0} / c\right) \partial_{z} j_{x}^{3 D}\left[n \ln \left(R_{J} / a_{0}\right)\right]^{3 / 2} \exp \left(-\pi n \ln \left(R_{J} / a_{0}\right)\left[4 T / s^{2}\left(\Phi_{0} / c\right) \partial_{z} j_{x}^{3 D}\right]^{2}\right)}{1-\operatorname{Erf}\left[4 T \sqrt{\pi n \ln \left(R_{J} / a_{0}\right)} / s^{2}\left(\Phi_{0} / c\right) \partial_{z} j_{x}^{3 D}\right]} .
$$

Expanding this, we obtain for small current gradients:

$$
\rho_{z}^{3 D}\left(\partial_{z} j_{x}^{3 D}\right)=\frac{T}{s j_{J}^{2}}\left(8 \pi D\left[n \ln \left(R_{J} / a_{0}\right)\right]^{2}+\frac{1}{4} n \ln \left(R_{J} / a_{0}\right) D\left[\frac{s^{2}}{T} \frac{\Phi_{0}}{c} \partial_{z} j_{x}^{3 D}\right]^{2}\right),
$$

i.e. a parabolic dependence on $\partial_{z} j_{x}^{3 D}$. On the other hand, for large current gradients,

$$
\rho_{z}^{3 D}\left(\partial_{z} j_{x}^{3 D}\right)=\frac{2 D}{s j_{J}^{2}}\left(\left[n \ln \left(R_{J} / a_{0}\right)\right]^{3 / 2} s^{2}\left(\Phi_{0} / c\right) \partial_{z} j_{x}^{3 D}+8\left[n \ln \left(R_{J} / a_{0}\right)\right]^{2} T\right)
$$

i.e., a linear dependence on $\partial_{z} j_{x}^{3 D}$.

As Eq. (44) is not convenient for analytical work, we will use an approximation of the form $\rho_{z}^{3 D}\left(\partial_{z} j_{x}^{3 D}\right)=$ $\rho_{0}^{3 D}+\sqrt{\left(\rho_{1}^{3 D}\right)^{2}+\left(f^{3 D} \partial_{z} j_{x}^{3 D}\right)^{2}}$ which gives a correct value at zero current gradient and the asymptotic behavior at large current gradients. It also approximates quite well the behavior of $\rho^{3 D}\left(\partial_{z} j_{x}^{3 D}\right)$ in the intermediate range of current gradients. Comparing the coefficients, we obtain

$$
\begin{aligned}
& \rho_{0}^{3 D}=\frac{16 D T}{s j_{J}^{2}}\left[n \ln \left(R_{J} / a_{0}\right)\right]^{2} \\
& \rho_{1}^{3 D}=(8 \pi-16) \frac{D T}{s j_{J}^{2}}\left[n \ln \left(R_{J} / a_{0}\right)\right]^{2} \\
& f^{3 D}=\frac{2 D s}{j_{J}^{2}} \frac{\Phi_{0}}{c}\left[n \ln \left(R_{J} / a_{0}\right)\right]^{3 / 2} .
\end{aligned}
$$




\section{B. Correlations between pancake positions}

Here we demonstrate how the results obtained above are modified in presence of inter- and intralayer correlations between pancake positions.

We first consider the effect of interlayer correlations. The presence of such correlations can be crudely described by regarding pancakes in different layers as tied together into vertical line segments of length $L_{z}$, which move as a whole. These segments should be used instead of independent pancakes of previous subsection. The phase differences $\delta \phi(\mathbf{r}, t)$ and corresponding Josephson currents are created only at the ends of these segments (more exactly, between layers, where one segment ends and another one starts), while the middle parts of the segments do not contribute to $\delta \phi(\mathbf{r}, t)$. This means, that the effective concentration of vortices is reduced by a factor $L_{z} / s$. Next, since each line segment has an increased "mass", the mobility $\mu$ and the diffusion constant $D$ are now reduced by another factor $L_{z} / s$. Finally, the vertical separation between the segments is $L_{z}$ instead of $s$ for free pancakes. This means that the velocity difference between the segments due to current gradient is increased by $L_{z} / s$. To take into account this and the reduction in the mobility in Eq.(43), the flux quantum $\Phi_{0}$ should be multiplied by $\left(L_{z} / s\right)^{2}$. This specifies, how the resistivity parameters are modified in the presence of interlayer correlations.

Next we turn to consider the intralayer correlations. Roughly speaking, these correlations cause pancakes in each layer to aggregate in clusters of size $L_{x y}$, so that there are $\left(L_{x y} / a_{0}\right)^{2}$ pancakes in a cluster. Pancakes inside each cluster are ordered, while different clusters move independently (actually, there is a hard-core repulsion between them). Since vortices in the same cluster are not independent, Eq.(34) for the phase correlation square now reads as

$$
\begin{aligned}
\left\langle S(\mathbf{r}, t)^{2}\right\rangle= & \sum_{\alpha}\left\langle\left(\sum_{i \in \alpha}\left[\mathbf{r}-\Delta \mathbf{R}_{1, i}(t)\right] \nabla \phi_{v}\left(-\mathbf{R}_{1, i}\right)\right)^{2}\right\rangle \\
& +\left\langle\left(\sum_{i \in \alpha}\left[\mathbf{r}-\Delta \mathbf{R}_{2, i}(t)\right] \nabla \phi_{v}\left(-\mathbf{R}_{2, i}\right)\right)^{2}\right\rangle,
\end{aligned}
$$

where $\alpha$ is an index of a cluster, while $i$ - of an individual pancake. For clusters which are far enough away, the differences in the location of individual pancakes inside the cluster can be neglected. Then each such cluster gives a contribution to $\left\langle S(\mathbf{r}, t)^{2}\right\rangle$, which is $\left(L_{x y} / a_{0}\right)^{4}$ times larger than a contribution of an individual pancake. On the other hand, the concentration of the clusters is $n\left(a_{0} / L_{x y}\right)^{2}$. To take both effects into account, we should multiply $n$ by $\left(L_{x y} / a_{0}\right)^{2}$ in the final result. Also, the diffusion constant (and the mobility) of each cluster is reduced by a factor $\left(L_{x y} / a_{0}\right)^{2}$, while the flux quantum $\Phi_{0}$ should be multiplied by the same factor. Substituting all these prescriptions into Eq.(47), we obtain the resistivity parameters in the presence of correlations between pancake positions:

$$
\begin{aligned}
\rho_{0}^{3 D} & =\frac{16 D T}{s j_{J}^{2}} \frac{\left(L_{x y} / a_{0}\right)^{2}}{\left(L_{z} / s\right)^{3}}\left[n \ln \left(R_{J} / a_{0}\right)\right]^{2} \\
\rho_{1}^{3 D} & =(8 \pi-16) \frac{D T}{s j_{J}^{2}} \frac{\left(L_{x y} / a_{0}\right)^{2}}{\left(L_{z} / s\right)^{3}}\left[n \ln \left(R_{J} / a_{0}\right)\right]^{2} \\
f^{3 D} & =\frac{2 D s}{j_{J}^{2}} \frac{\left(L_{x y} / a_{0}\right)^{3}}{\left(L_{z} / s\right)^{1 / 2}} \frac{\Phi_{0}}{c}\left[n \ln \left(R_{J} / a_{0}\right)\right]^{3 / 2} .
\end{aligned}
$$

Here we neglected all changes in the argument of the logarithms.

\section{Transformation of the parameters into 1D form}

Now we transform these quantities into a form appropriate for the $1 \mathrm{D}$ macroscopic model. For this, we first define the corresponding fields and currents from their $3 \mathrm{D}$ counterparts (assuming that everything is uniform in $\hat{\mathbf{y}}$ direction):

$$
\begin{aligned}
& j_{x}(z)=j_{x}^{3 D}\left(x=L_{x} / 2, z\right) L_{y} \\
& J_{z}(z)=\int_{0}^{L_{x} / 2} d x j^{3 D}(x, z) L_{y} \\
& V(z)=\int_{0}^{L_{x}} d x E_{x}^{3 D}(x, z) \\
& E_{z}(z)=E_{z}^{3 D}(x=0, z),
\end{aligned}
$$

where $L_{x}$ and $L_{y}$ are sizes of the sample. Then, for large current gradients, we use the Ohm's law for the 3D sample and average over $\mathrm{x}$ :

$$
\begin{aligned}
\int_{0}^{L_{x} / 2} d x E_{x}^{3 D}(x, z)= & \int_{0}^{L_{x} / 2} d x \rho_{x}^{3 D} j_{x}^{3 D}(x, z) \\
\int_{0}^{L_{x} / 2} d x E_{z}^{3 D}(x, z)= & \int_{0}^{L_{x} / 2} d x\left(\rho_{0}^{3 D} j_{z}^{3 D}(x, z)\right. \\
& \left.+f^{3 D} \partial_{z} j_{x}^{3 D}(x, z) j_{z}^{3 D}(x, z)\right) .
\end{aligned}
$$

Defining now the reduced quantities as ratios between the $3 \mathrm{D}$ and $1 \mathrm{D}$ ones, so that

$$
\begin{aligned}
j_{x}^{r e d}(x, z) & \equiv \frac{j_{x}^{3 D}(x, z)}{j_{x}(z)} \\
j_{z}^{r e d}(x, z) & \equiv \frac{j_{z}^{3 D}(x, z)}{J_{z}(z)} \\
E_{x}^{r e d}(x, z) & \equiv \frac{E_{x}^{3 D}(x, z)}{V(x, z)} \\
E_{z}^{r e d}(x, z) & \equiv \frac{E_{z}^{3 D}(x, z)}{E_{z}(z)},
\end{aligned}
$$

we obtain from the previous equations: 


$$
\begin{aligned}
V(z) \int_{0}^{L_{x} / 2} d x E_{x}^{r e d}(x, z)= & j_{x}(z) \rho_{x}^{3 D} \int_{0}^{L_{x} / 2} d x j_{x}^{r e d}(x, z) \\
E_{z}(z) \int_{0}^{L_{x} / 2} d x E_{z}^{r e d}(x, z)= & J_{z}(z) \rho_{0}^{3 D} \int_{0}^{L_{x} / 2} d x j_{z}^{r e d}(x, z) \\
& +J_{z}(z) f^{3 D} \int_{0}^{L_{x} / 2} d x j_{z}^{r e d}(x, z) \partial_{z} j_{x}(z) j_{x}^{r e d}(x, z) .
\end{aligned}
$$

Then, in order to obtain the equations of the macroscopic model, we make two assumptions: first, we neglect the derivative $\partial_{z} j_{x}^{r e d}(x, z)$; second, we assume that the reduced quantities are not affected by shear effects, so we calculate them from a linear model with $f=0$. The parameters of the macroscopic $1 \mathrm{D}$ model are then given by

$$
\begin{aligned}
R_{x} & =\rho_{x}^{3 D} \int_{0}^{L_{x} / 2} d x j_{x}^{r e d}(x, z) / \int_{0}^{L_{x} / 2} d x E_{x}^{r e d}(x, z) \\
\rho_{0} / 2 & =\rho_{0}^{3 D} \int_{0}^{L_{x} / 2} d x j_{z}^{r e d}(x, z) / \int_{0}^{L_{x} / 2} d x E_{z}^{r e d}(x, z) \\
f / 2 & =f^{3 D} \int_{0}^{L_{x} / 2} d x j_{z}^{r e d}(x, z) j_{x}^{r e d}(x, z) / \int_{0}^{L_{x} / 2} d x E_{z}^{r e d}(x, z),
\end{aligned}
$$

where $\rho_{0}$ and $f$ are divided by 2 , since, as we explained in the beginning of the previous section, the perpendicular resistivity of the macroscopic model is taken to be $\rho_{z} / 2$.

To find the reduced quantities, we need to find the current distribution in a sample with constant resistivities $\rho_{x}^{3 D}$ and $\rho_{z}^{3 D}$. This amounts to solving the Laplace equation with the boundary conditions $j_{x}^{0}(x=0, z)=j_{x}^{0}(x=$ $\left.L_{x}, z\right)=j_{z}^{0}(x, z=\infty)=0$, and $j_{z}^{0}(x, z=0)=0$, except two narrow regions near $x=0$ and $x=L_{x}$, where $j_{z}^{0}(x, z=0)$ is, respectively, positive and negative. This describes contacts, attached to the top of the sample, where the current flows into and out of the system. For simplicity we assumed here that the system is infinitely thick in $\hat{\mathbf{z}}$ direction. Choosing an appropriate form for $j_{z}^{0}(x, z=0)$, we obtain:

$$
\begin{aligned}
j_{z}^{0}(x, z) & =\frac{\sinh k_{0}(w+\alpha z) \cos k_{0} x}{\sinh ^{2} k_{0}(w+\alpha z)+\sin ^{2} k_{0} x} \\
j_{x}^{0} & =\alpha \frac{\sin k_{0} x \cosh k_{0}(w+\alpha z)}{\sinh ^{2} k_{0}(w+\alpha z)+\sin ^{2} k_{0} x},
\end{aligned}
$$

where $w$ is the width of the contacts $\left(w \ll L_{x}\right), \alpha \equiv$ $\sqrt{\rho_{z}^{3 D} / \rho_{x}^{3 D}}$, and $k_{0} \equiv \pi / L_{x}$. Using this, we calculate the integrals of the reduced quantities and substitute them into Eq. (54), thus obtaining

$$
\begin{aligned}
R_{x} & =\rho_{x}^{3 D} \frac{2 L_{x}}{\pi L_{y}} \cosh k_{0}(w+\alpha z) \log \operatorname{coth} k_{0}(w+\alpha z) / 2 \\
\rho_{0} / 2 & =\rho_{0}^{3 D} \frac{\pi}{L_{x} L_{y}} \frac{1}{\sinh k_{0}(w+\alpha z) \arctan 1 / \sinh ^{2} k_{0}(w+\alpha z)} \\
f / 2 & =f^{3 D} \frac{\pi}{2 L_{x} L_{y}^{2}} \frac{1}{\sinh ^{2} k_{0}(w+\alpha z) \arctan ^{2} 1 / \sinh k_{0}(w+\alpha z)} .
\end{aligned}
$$

Here a finite $z$ should be taken, so that $k_{0} \alpha z \propto 1$. Then the hyperbolic functions give factors of order 1 , and the $1 \mathrm{D}$ parameters are given by

$$
\begin{aligned}
R_{x} & =\rho_{x}^{3 D} \frac{2 L_{x}}{\pi L_{y}} \\
\rho_{0} / 2 & =\rho_{0}^{3 D} \frac{\pi}{L_{x} L_{y}} \\
f / 2 & =f^{3 D} \frac{\pi}{2 L_{x} L_{y}^{2}} .
\end{aligned}
$$

This establishes a correspondence between 3-dimensional resistivity parameters and the 1-dimensional ones, which were used in the macroscopic model.

\section{DISCUSSION AND CONCLUSIONS}

It is not easy to compare directly predictions of our model with the experimental results, since we do not know temperature dependence of various parameters of 
the model. Hence we make only qualitative statements based on robust features of the model.

First, the model predicts that the resistance grows with the current (at least for not too small currents), and this current non-linearity becomes very strong as $J_{i n} \rightarrow J_{0}$. This is consistent with the experimental result. Using the results of the microscopic calculation Sec. III, we found that without correlations between the pancakes $J_{0}$ is much larger than the relevant $J_{i n}$. However, in presence of correlations its value is suppressed by a factor of $\left(L_{x y} / a_{0}\right)\left(L_{z} / s\right)^{5 / 2}$, thus making its value much closer to $J_{i n}$. If the ratios $L_{x y} / a_{0}, L_{z} / s$ are assumed to be 10-15, $J_{0}$ becomes comparable with the experimentally relevant currents. This provides an explanation to the experimental fact that the current non-linearity becomes strong below the depinning transition temperature $T_{d}$, where correlations between the pancakes start to build up. Next, the model explains the feature of re-entrance, that is, the experimental observation that below the depinning transition the resistance increases as the temperature is decreased. According to the model, if the interface resistance $R_{\text {int }}$ is large enough, the resistance of the system grows with $j_{c}$, which naturally starts to grow as the temperature is decreased below $T_{d}$. Moreover, the model predicts that this rise in the resistance should be more pronounced for smaller currents, as indeed observed.

Some ingredients are missing from our model. First, the model approximates $R_{x}$ to be independent of the intra-layer current. This approximation is presumably good above the depinning temperature, but becomes poor below that temperature, where intra-layer current induces vortex depinning. Second, a missing ingredient in our work is a microscopic derivation of the interface resistance $R_{\text {int }}$, separating between the resistive and nonresistive parts of the sample. The microscopic origin we have in mind is that in the region between the two phases the pancake mobility is very sensitive to parallel current variation. Then a small current gradient is enough to create a large pancake velocity gradient, which would cause a large perpendicular resistance in that region. Our attempts to provide a microscopic derivation of $R_{\text {int }}$ and its temperature dependence led us to results that heavily depend on various microscopic parameters whose values and temperature dependences are not known. We were therefore led to leave $R_{\text {int }}$ as a phenomenological parameter.

Altogether, then, our work is able to explain the qualitative features of the non-linear transport observed in Ref. 1 and unravel a unique feature of transport in super- conducting BSCCO samples in perpendicular magnetic field.

\section{ACKNOWLEDGMENTS}

We thank E. Zeldov and V. Geshkenbein for useful discussions. This work is supported by the Israel Science Foundation and the Victor Ehrlich chair.

${ }^{1}$ B. Khaykovich, D. T. Fuchs, K. Teitelbaum, Y. Myasoedov, E. Zeldov, T. Tamegai, S. Ooi, M. Konczykowski, R. A. Doyle, and S. F. W. R. Rycroft, Phys. Rev. B 61, R9261 (2000).

${ }^{2}$ R. Busch et al., Phys. Rev. Lett. 69, 522 (1992).

${ }^{3}$ H. Safar et al., Phys. Rev. B 46, 14238 (1992).

${ }^{4}$ J. H. Cho et al., Phys. Rev. B 50, 6493 (1994).

${ }^{5}$ L. N. Bulaevskii, M. Ledvij, and V. G. Kogan, Phys. Rev. B 46, 366 (1992).

${ }^{6}$ C. P. Bean, Phys. Rev. Lett. 8, 250 (1962).

${ }^{7}$ E. H. Brandt, Rep. Prog. Phys. 58, 1465 (1995).

${ }^{8}$ E. Zeldov, J. R. Clem, M. McElfresh, and M. Darwin, Phys. Rev. B 49, 9802 (1994).

${ }^{9}$ K. E. Gray and D. H. Kim, Phys. Rev. Lett. 70, 1693 (1993).

10 A. E. Koshelev, Phys. Rev. Lett. 76, 1340 (1996).

${ }^{11}$ G. Briceño, M. F. Crommie, and A. Zettl, Phys. Rev. Lett. 66, 2164 (1991).

12 A. Yurgens et al., Phys. Rev B 59, 7196 (1999).

${ }^{13}$ D. Thopart, Ch. Goupil, and Ch. Simon, Phys. Rev. B 63, 184504-1 (2001).

${ }^{14}$ L. I. Glazman and A. E. Koshelev, Phys. Rev B 43, 2835 (1991).

15 J. R. Clem, Phys. Rev B 43, 7837 (1991).

${ }^{16}$ L. L. Daemen, L. N. Bulaevskii, M. P. Maley, and J. Y. Coulter, Phys. Rev. B 47, 11291 (1993).

${ }^{17}$ G. Blatter et al., Rev. of Mod. Phys. 66, 1125 (1994).

18 A. E. Koshelev, L. I. Glazman, and A. I. Larkin, Phys. Rev. B 53, 2786 (1996).

19 A. E. Koshelev, P. Le Doussal, and V. M. Vinokur, Phys. Rev B 53, R8855 (1996).

${ }^{20}$ L. N. Bulaevskii, M. P. Maley, and V. M. Vinokur, Phys. Rev B 57, R5626 (1998).

${ }^{21}$ N. Morozov et al., Phys. Rev. Lett. 82, 1008 (1999).

${ }^{22}$ N. Morozov et al., Phys. Rev. B 60, 96 (1999). 